\title{
Systematic review of the effect of individual and combined nutrition and exercise interventions on weight, adiposity and metabolic outcomes after delivery: evidence for developing behavioral guidelines for post-partum weight control
}

\author{
Alexander Arkin Berger ${ }^{1}$, Rachel Peragallo-Urrutia ${ }^{2}$ and Wanda K Nicholson ${ }^{3,4^{*}}$
}

\begin{abstract}
Background: Post-partum weight retention contributes to the risk of chronic obesity and metabolic alterations. We conducted a systematic review of randomized controlled trials (RCTs) on the effect of post-partum nutrition and exercise interventions on weight loss and metabolic outcomes. DATA SOURCES: Four electronic databases were searched from inception to January, 2012. Two investigators reviewed titles and abstracts, performed data abstraction on full articles and assessed study quality.

Methods: We included RCTs comparing nutrition, exercise or combined nutrition and exercise interventions with a control condition. Thirteen studies met our inclusion criteria ( $N=1,310$ participants). Data were abstracted on study characteristics, intervention components, enrollment period, and length of follow-up. Outcomes of interest included weight, adiposity, cardio-metabolic measures (glucose, lipids) and obesity-related inflammatory markers.

Results: Nine trials compared combined interventions to standard post-partum care; three trials assessed the effect of exercise interventions, one trial evaluated a nutrition-only intervention. Four good quality RCTs on combined interventions had inconsistent findings, with the larger RCT $(N=450)$ reporting no difference in weight between groups. Four fair-to good quality RCTs reported greater weight loss in the combined intervention group vs. standard care, ranging from $0.17 \mathrm{~kg}$ to $4.9 \mathrm{~kg}$. Results from exercise only interventions were inconclusive. Evidence for nutrition only interventions was insufficient. There was insufficient evidence for the effect of post-partum interventions on metabolic risk factors and inflammatory biomarkers.

Conclusions: Combined nutrition and exercise interventions can achieve weight loss, but evidence is limited due to a small number of trials and limitations in study design.
\end{abstract}

Keywords: Systematic review, Obesity, Post-partum weight retention, Randomized trials

\footnotetext{
* Correspondence: wknichol@med.unc.edu

${ }^{3}$ Department of Obstetrics \& Gynecology, Division of Women's Primary Health, 3027 Old Clinic Building, CB \# 7570, Chapel Hill, NC, USA

${ }^{4}$ Diabetes and Obesity Core, Center for Women's Health Research, University of North Carolina School of Medicine, 3027 Old Clinic Building, CB \# 7570

Chapel Hill, NC, USA

Full list of author information is available at the end of the article
} 


\section{Background}

Obesity, fueled largely by excessive caloric intake and sedentary lifestyles, contributes to multiple adverse health conditions in women, including diabetes, cardiovascular disease, and certain cancers (endometrial, colon, and postmenopausal breast). More than 78 million adults age 20 years and older in the United States are obese; 41 million are women [1]. The post-partum period may be a critical period for long-term weight gain and development of obesity for women [2,3]. Physiological changes of childbirth may contribute to weight retention and weight gain $[4,5]$. Compared with weight gain during other life intervals, excess weight retained after childbirth appears to be particularly harmful, as evidence suggests that weight retained in the post-partum tends to be distributed centrally, and therefore, may increase the risk of developing chronic disease $[2,6]$. Post-partum interventions that target dietary intake and exercise may help women to achieve a healthy weight after delivery and improve their overall health status.

Interventions that integrate exercise or dietary changes have been shown to achieve weight loss, and reduce cardiovascular [7] and diabetes-related risk factors [8] in middle- and older-aged women, though evidence for their efficacy in post-partum women is limited. By reducing weight after delivery, women may be able to enter a subsequent pregnancy at a healthier weight and reduce their risk of obesity-related pregnancy complications [9-11]. Alternatively, if a woman has completed childbearing, achieving a healthy weight after delivery may help to improve long-term health through the effects on metabolic and obesity-related biomarkers. The American Congress of Obstetricians and Gynecologists [12] provides general recommendations for post-partum physical activity, but there are no current evidence-based U.S. guidelines for diet or exercise interventions to help women achieve a healthy weight after delivery and improve.

A recent systematic review of eight studies [13], suggests that diet and exercise interventions can reduce postpartum weight retention. However, there was no assessment of the effects of lifestyle interventions on metabolic risk factors, such as glucose and lipid levels or adipokines. Prior research suggests that weight loss improves longterm health outcomes through favorable effects on glucose metabolism, lipid aberrations and adipokines. Adipocytokines, such as adiponectin and C-reactive protein, and leptin are inflammatory proteins that have been linked to obesity and type 2 diabetes [14-17]. Adiponectin levels are lower in subjects with obesity and type 2 diabetes; leptin and c-reactive protein correlate positively with body mass index, insulin resistance and diabetes [18,19]. Elucidating the effects of lifestyle modification on these biomarkers is important to perinatal health providers as it can help them to better inform patients about the potential benefits of postpartum weight loss programs. Additionally, there was little to no data on potential harms of post-partum diet or exercise interventions. The RCTs included in the review were largely limited to combined diet and exercise interventions. Relevant data on the effects of diet and/or exercise on weight and the effects of weight change on obesity-related biomarkers is needed to foster the development of evidence based guidelines for weight loss in the postpartum period.

We conducted a systematic review of the literature to identify randomized trials to assess the benefits and harms of post-partum behavioral weight management interventions that included nutrition, exercise, or combined nutrition and exercise components. The primary questions were 1) Do post-partum behavioral weight management interventions (nutrition, exercise or combined nutrition and exercise) lead to a reduction in weight, body mass index (BMI) or adiposity in women who were overweight or obese at the time of conception or in early pregnancy? 2) Do post-partum behavioral weight management interventions lead to other positive outcomes (e.g. improved glucose and insulin, levels of inflammatory biomarkers)? and 3) what are the adverse effects of post-partum behavioral weight management interventions attempting to reduce weight and improve health outcomes?

\section{Methods}

A preliminary protocol was designed and independently reviewed. The protocol included the literature search strategy, the selection process for choosing studies, the method and forms to be used for extracting data, assessing the quality of studies and the methods for data synthesis and analysis.

\section{Criteria for considering studies for this review Types of studies}

We considered clinical trials that met our pre-determined list of inclusion criteria that were reviewed and approved as part of the preliminary protocol. We included trials that had a least one intervention and one comparison group, which could include a control group, usual care group or alternative behavioral intervention group. Studies without a comparison group were not included.

\section{Types of participants}

We included studies which enrolled participants with the following factors: women who were postpartum, enrolled in the study in the early postpartum period (up to 12 weeks following delivery); women from any geographical and racial/ethnic background. 


\section{Types of interventions}

Interventions that included diet, exercise or diet plus exercise components, took place in the postpartum period and were of at least 4 weeks in duration with the goal of reducing postpartum weight were included in this review. We only considered interventions that included behavioral modifications. Studies that focused on weight loss medications or surgical therapy (e.g. bariatric surgery) for weight loss were not included in the review. Interventions could be conducted in any setting, including primary care settings and hospitals or in community settings.

\section{Types of outcome measures}

The primary outcome was weight. Additional outcomes of interest for weight and adiposity included percentage of weight loss, proportion of women returning to prepregnancy weight, and measures of adiposity, including abdominal (waist) circumference [20], waist-to-hip ratio, and skinfold thickness, and any reported adverse events of the intervention. Cardio-metabolic outcomes included lipids high-density lipoprotein cholesterol [HDL-C], lowdensity lipoprotein cholesterol [LDL-C], triglycerides), glucose and glycosylated hemoglobin (HbA1c). Obesityrelated biomarkers, such as c-reactive protein, interleukin6 , leptin and adiponectin.

\section{Search methods for identification of studies}

A comprehensive search strategy was developed (Search strategy in Additional file 1). We searched 4 electronic databases, MEDLINE, EMBASE, The Cochrane Central Register of Controlled Trials, and the Cumulative Index to Nursing and Allied Health Literature for English-only articles from inception through January 2012. Search terms included $\mathrm{MeSH}$ and text terms for post-partum, diet and exercise, behavioral interventions and weight loss. The search strategy included selected index terms, key words and free text terms such as:

"Postpartum period*, postpartum, post partum, post pregnancy, intervention, behavior, "behavioral, life style", lifestyle, life style, "Exercise Therapy", exercise ${ }^{*}$, diet* or exercise therapy* or exercise ${ }^{*}$

We conducted quality audits to confirm that studies identified through prior literature review and discussions with experts in the field were identified by the search terms. We hand-searched 15 journals that were most likely to publish articles on post-partum weight loss interventions from December 2011 through January 2012 and scanned reference lists from included articles and relevant review articles. Subsequently, we conducted a bridge search from January, 2012 to May, 2013 (See PRISMA checklist in Additional file 2).

\section{Data collection and analysis Selection of studies}

Articles were rejected if they met one or more of the following exclusion criteria: 1) not a RCT; 2) not written in English; 3) did not include human data; 4) no original data (i.e. meeting abstract, editorial, commentary or letter); 5) did not include data on maternal weight loss; 6) less than $50 \%$ of the sample was enrolled within one year post-partum; 7) did not compare a diet, exercise or combined diet and exercise intervention to a control group; and 8) intervention was less than 4 weeks in duration. When a title/abstract could not be rejected with certainty, the full text of the article was obtained for further evaluation. After non-relevant studies were excluded, potentially relevant studies were assessed independently by two reviewers (AB, RPU). Differences between reviewers' assessments were resolved by discussion and consensus.

\section{Data extraction and management Data synthesis}

Two reviewers sequentially abstracted information from each articles using standardized forms. Reviewers abstracted information regarding study characteristics, participants, study setting (for example, city or geographical region), study participants (for example, age, race), intervention type (diet, exercise or combinations of each), mode of intervention delivery (for example, mail correspondence, in-person), timing of initiation of the postpartum intervention, duration of the intervention, length of follow-up after the intervention. Differences were resolved through discussion.

\section{Assessment of study quality, bias and evidence grading}

Two investigators (AB, RPU) independently assessed the quality of trials and used items for selection bias, treatments, outcome measures, statistical methods, and loss to follow-up using methods from the United States Preventive Services Task Force [21,22] and the University of York Centre for Reviews and Dissemination [23]. The quality of each study was rated as good (low risk for bias), fair, or low (high risk for bias).

The quantity, quality, and consistency of the results, the directness of the measures used for each outcome; the precision of the results and the magnitude of effects were graded based on the GRADE (The Grading of Recommendations Assessment, Development and Evaluation) working group criteria [24]. "High" strength of evidence indicates that the evidence probably reflects the true effect, "moderate" strength indicates that further research may change the results, and "low" strength indicates low confidence that the evidence reflects the true effect and further research is very likely to change the result. "Insufficient" evidence indicates that no studies met 
our inclusion criteria for the comparison for a given outcome.

\section{Data synthesis}

We conducted a qualitative summary for each outcome by intervention category (diet and exercise, exercise-only and diet-only). We were unable to conduct a meta-analysis of the primary outcome of weight or any secondary outcomes because of heterogeneity in the population studied, variation in study duration and intervention components, and mode of delivery of the intervention components.

\section{Results}

\section{Results of the search}

We retrieved a total of 122 unique citations from electronic databases, hand-searching, and a bridge search (Figure 1). After abstract and full article review, we identified 13 unique publications related to the benefits and harms of behavioral interventions for post-partum weight loss; nine RCTs assessed the effects of combined diet and exercise interventions, ( $\mathrm{N}=1,015$ participants); three RCTs compared exercise-only interventions to usual care $(\mathrm{N}=139$ participants); one RCT compared a dietonly intervention to usual care ( $\mathrm{N}=151$ participants $)$.

\section{Description of 13 included trials}

Thirteen RCTs were published between 1998 and May 2013 and were conducted in various countries (Table 1).
Nine trials were conducted in the United States; one in Taiwan [25], one in Iran [26], one in Greece [27] one in Sweden [20] and one in the United Kingdom [28]. The trials also compared interventions using different modes of delivery. Among the trials of combined interventions, 3 RCTs compared an in-person dietary and exercise intervention to usual care [20,29]. Bertz and colleagues [30], provided an in-person intervention with dietary modifications aimed at reducing caloric intake by $500 \mathrm{kcal} /$ day and complete 45 minutes of exercise. Huang [25] provided individualized diet and exercise plans. Ostbye and coauthors [29] provided an in-person intervention coupled with telephone calls. Three RCTs compared in-person nutrition and exercise interventions to usual care plus an informational pamphlet $[28,31]$ or structured handout [32]. One trial [33] compared a diet plus moderate or low intensity in-person intervention with historical controls.

There were exercise-only RCTs $[26,27,34]$. One trial included in-person structured aerobic and strength training sessions [27]. A second RCT included a pedometer-based walking program with text reminders and telephone feedback [26]. Another trial consisted of individually tailored and in-person supervised aerobic training [34]. A nutritiononly trial [35] compared the effect of individual nutritional counseling and facilitated group sessions with standard post-partum care.

The enrollment period for the 13 trials ranged from one day to six months after delivery. The duration of the

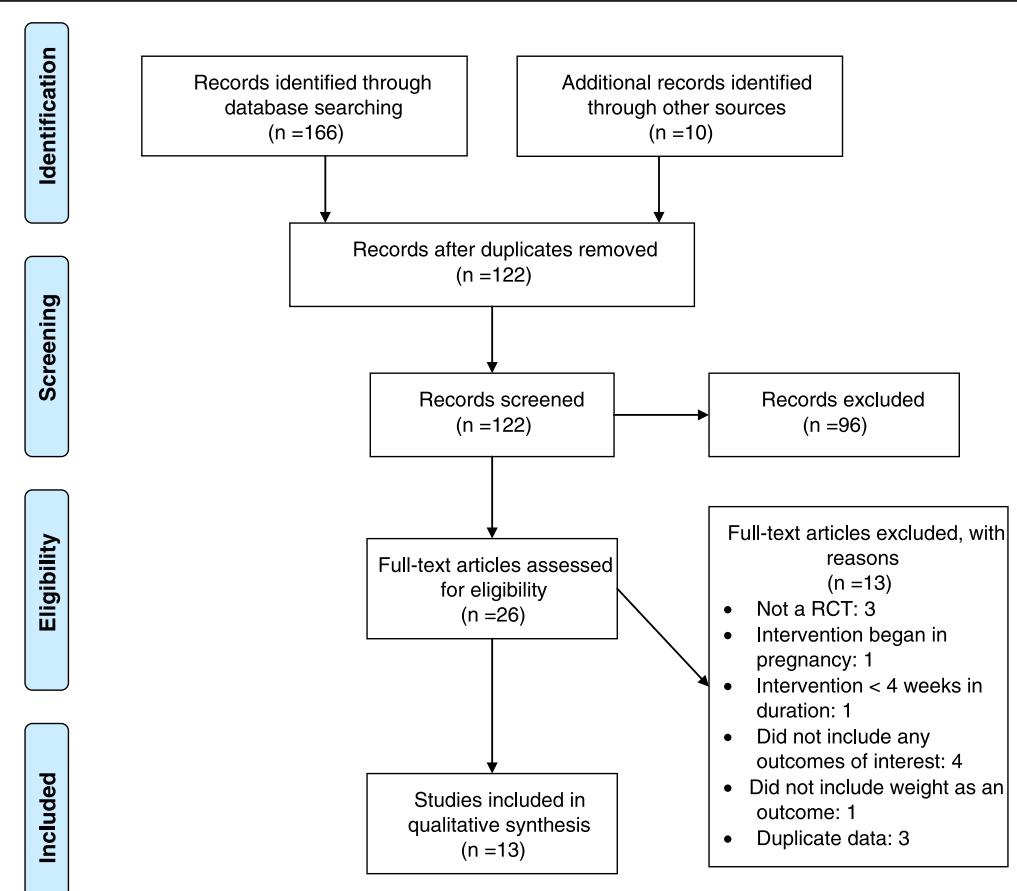

Figure 1 Summary of the literature search and review process (number of articles). *Total may exceed the number in the corresponding box because articles could be excluded for more than one reason at this level. CINAHL (Cumulative Index to Allied Health and Nursing Literature). 
Table 1 Characteristics of 13 RCTs of postpartum nutrition, exercise and combined interventions

Author, yr, Country Description of treatment arms
in-person [30]

Colleran, 2012,US, Intervention (I): postpartum counseling on diet and in-person [32] exercise plus on-line resources; Control (C): Usual

Leermakers 1998, US, Intervention (I): Correspondence lessons, group

in-person [31]

O'Toole, 2003 US,

in-person [36]

Craigie 2011, United I: Information pamphlet + two face to face counseling Kingdom, in-person [28] sessions, with telephone follow-up for reinforcement

Huang, 2011, Taiwan, I: Individualized dietary and physical activity plans, in-person [25] including 6 pregnancy sessions and 3 postpartum

Lovelady 2000, US, I: Caloric restriction and exercise intervention, including in-person [37] 4 exercise sessions, lasting 43 minutes with goal of

Ostbye, 2009, US, I: 8 healthy eating classes, 10 physical activity classes

in-person [29]

Davenport, 2011, US, I: Diet + low intensity exercise

in-person [33]

\section{Exercise-only intervention}

Zourladani, 2011, I: Instructor led 1-hour exercise class with aerobic activity Greece [15] and strength training 3 times per wk for 12 wks.

Maturi, 2011, Iran [14]

(1): 3 intervention arms and 1 control arm (D) dietary modifications reduce caloric intake by $500 \mathrm{Kcal} / \mathrm{day} ;$ (E): exercise only- exercise to achieve
$45 \mathrm{~min}$ brisk walk $4 \mathrm{~d} /$ week; (DE): combined diet and exercise; Control (C): Usual care care + diet and nutrition print handouts sessions and telephone follow-up Control (C): Usual care + informational brochure

: Structured diet + exercise with weekly in-person sessions $\times 12$ wks, bi-weekly sessions $\times 8$ wks, and monthly sessions up to 1-year

C: One in-person session followed by self-directed program and resources C: Information pamphlet sessions C: Usual care $65-80 \%$ heart rate C: Usual care and 6 telephone counseling sessions over 9 mos. C: Usual care

I: Diet + moderate intensity exercise

C: Historical controls matched for age, BMI, and parity

$4-6$ wks; 3 mos

C: No intervention

\section{Enrollment period; Sample population,}

* duration

10-14 weeks

postpartum;

12 weeks

63 lactating, overweight/obese

primiparous; $\mathrm{N}=68$

4-6 weeks:16 weeks

31 women, $\geq 23$ years and $<37$ years, singleton pregnancy

$(-5.8+3.5 \mathrm{~kg})(7 \%)$ compared with the $C(-1.6+5.4 \mathrm{~kg})(3 \%)(P=0.03)$

8 mos; 6 mos

Non-lactating postpartum women; $\mathrm{N}=90$

6 wks-6 mos;

6-10 mos

Postpartum women who were overweight

or obese prior to pregnancy; $\mathrm{N}=40$

6-18 mos; 3 mos

Low income, overweight and obese postpartum women; $\mathrm{N}=52$

Age Race/ethnicity

Study

quality

33 Swedish

Good

1 day; 6 mos

Pregnant and postpartum women Taiwanese 32 women; 1 day postpartum; $\mathrm{N}=189$

5 wks; 2.5 mos

2 mos; 9 mos

8 wks; 4 mos

$6 w k s-6 m o s ; 3$ mos
Overweight postpartum women with BMl $25-30 \mathrm{~kg} / \mathrm{m} 2$, exclusively breastfeeding;

$\mathrm{N}=40$

Overweight or obese postpartum women $N=450$

Overweight or obese women who

I: $45 \%$ AA

C: $45 \%$ AA

Intervention groups: Fair-to-good $85-90 \%$ white

No AA

Other race: NR

Primiparous postpartum women $\mathrm{N}=40$

Gree

Fair

actating, normal or overweight postpartum women; $\mathrm{N}=66$ retained $>5 \mathrm{~kg}$ after delivery; $\mathrm{N}=60$
Fair

Fair-to-good

Fair-to-good

Good 
Table 1 Characteristics of 13 RCTs of postpartum nutrition, exercise and combined interventions (Continued)

\begin{tabular}{|c|c|c|c|c|c|c|}
\hline & $\begin{array}{l}\text { Tailored pedometer-based walking program with baseline } \\
\text { counseling session, cell phone and text reminders and } \\
\text { telephone feedback; C: Usual Care }\end{array}$ & & & & & \\
\hline \multirow[t]{2}{*}{ Dewey, 1994, US [34] } & $\begin{array}{l}\text { I: Individually tailored and supervised aerobic activity to } \\
\text { achieve } 60-70 \% \text { heart rate reserve. } 45 \text { min- } 5 \text { times a wk }\end{array}$ & 6-8 wks; 3 mos & $\begin{array}{l}\text { Exclusively breastfeeding postpartum } \\
\text { women; } N=33\end{array}$ & 30 & No AA & Fair-to-good \\
\hline & C: No intervention & & & & & \\
\hline \multicolumn{7}{|c|}{ Nutrition-only Interventions } \\
\hline \multirow[t]{2}{*}{ Krummel, 2010, US [23] } & $\begin{array}{l}\text { I: Counseling with dietitian, and } 10 \text { facilitated discussion } \\
\text { groups, monthly personalized feedback on self-monitoring } \\
\text { records }\end{array}$ & 30 wks; 12 mos & $\begin{array}{l}\text { Postpartum women enrolled in WIC; } \\
N=151\end{array}$ & 27 & 10\% non-white; & Fair-to-poor \\
\hline & C: Self directed & & & & & \\
\hline
\end{tabular}

*Postpartum enrollment period; **duration of postpartum intervention. NR = not reported; BMI = body mass index; AA = African American; mos = months; wks = weeks; WIC = Women's, Infant, Children's program. 
interventions was 3 to 9 months. Among the nine trials comparing combined diet and exercise interventions [20,25,28,32,33,36-38] with standard care, attrition rates varied widely. Two of these trials [31,37] reported differential loss to follow up between the intervention and standard care groups. Two trials did not discuss attrition rates [25,28]. For the exercise-only studies [26,27,34], loss to follow-up rates ranged from $5-13 \%$.

\section{Quality of included trials}

There were three good quality RCTs [20,36,38] with broad applicability to the general population of postpartum women. There were six fair-to-good quality trials $[27,31,32,34,35,37]$. Only three trials $[28,31,38]$ discussed or reported an intention to treat analysis $[31,38]$.

\section{Results of included trials}

\section{Nutrition and exercise interventions}

Nine trials compared the effects of post-partum of diet and exercise interventions compared to standard postpartum care on weight (Table 2). Seven of the trials $[20,28,32,33,36,37]$ reported greater post-partum weight loss among women in the intervention group compared to those in the usual care group. One trial compared a diet-only, exercise-only and combined diet and exercise intervention to usual care [20]. Ostbye and colleagues [39] reported findings from the largest RCT, in which they randomly assigned 450 post-partum women to receive a combined diet and exercise intervention or standard care. They reported no statistically significant differences in mean weight loss between the two groups. Two trials $[33,37]$ evaluated body mass index (BMI) and reported a statistically significant difference between the intervention and standard care groups. Leermakers and colleagues [31] found a statistically significantly higher percentage weight loss $(10 \%$ versus $5.8 \%$; $<<0.04)$ and proportion of women returning to their pre-pregnancy weight $(33 \%$ versus $11.5 \%, \mathrm{p}<0.05)$ (data not shown) in the intervention group compared to the standard care group. There were no statistically significant differences in abdominal circumference between women in a diet and exercise intervention and those in usual care.

In a 3-arm RCT, Davenport and colleagues [33] reported outcomes among participants completing a nutrition + low intensity intervention and a nutrition + moderate intensity to historical controls matched for age, BMI and parity. There was statistically significant greater weight loss $(-5.0 \pm 2.9$ moderate intensity, $-4.2 \pm 4.0$ low intensity, respectively, compared to controls $-0.1 \pm 3.3$; $\mathrm{P}<0.01)$ and lower waist-to-hip ratios $(-0.05$ moderate intensity, -0.03 low intensity vs. 0.00 control; $\mathrm{P}<0.05)$ among participants in the intervention groups compared to historical controls matched for age, BMI and parity (Table 2). Davenport and co-investigators [33] also reported on differences in cardio- metabolic and biomarker outcomes between the three groups. There were small, but statistically significantly lower fasting glucose (I: $-0.07 \mathrm{mg} / \mathrm{dl}, \mathrm{I}:-0.5 \mathrm{mg} / \mathrm{dl}$, and C: $0.7 \mathrm{mg} / \mathrm{dl} ; \mathrm{P}, 0.05$ ) and adiponectin (I: 3,610 ng/ml, :3,890 ng/ml, C: $1575 \mathrm{ng} / \mathrm{ml} ; \mathrm{P}<0.05)$ levels in the nutrition plus moderate and nutrition plus low intensity exercise intervention groups, respectively, compared to historical controls. There were no available data on $\mathrm{C}$ reactive protein or leptin levels.

\section{Exercise-only interventions}

Maturi and colleagues [26] reported greater weight loss in the intervention group compared to the usual care group. Also, women in the intervention group had a lower BMI $(\mathrm{p}<0.001)$ and waist-to-hip ratio $(\mathrm{p}=0.02)$ compared to those in the usual care group. Two smaller RCTs [27,34] reported no statistically significant differences in average weight loss. Maturi and colleagues [26] reported waist-tohip ratios that were lower among women receiving an individualized pedometer-based program compared to those in usual care.

\section{Nutrition-only interventions}

One trial [23] met our inclusion criteria. Krummel and colleagues [35] reported no difference in weight or BMI between women in a diet-only intervention and those receiving usual care.

\section{Adverse effects of post-partum behavioral interventions}

We found little data on harms of post-partum behavioral interventions. There were no reports of adverse maternal events or deaths related to the interventions. Dewey and colleagues reported no change in milk volume and composition among women enrolled in an exercise-only intervention compared to usual care [34].

\section{Overall strength of evidence}

Taking into account the number of RCTs, study quality inconsistency in outcome measures across studies and inconsistent study findings, we graded the overall strength of evidence as low for the effect of post-partum lifestyle interventions on the reported maternal outcomes (Table 3). The duration of interventions was generally short ( $\leq 9$ months). Little to no information was provided on treatment harms or adverse events.

\section{Discussion}

We found an overall low strength of evidence for postpartum interventions on weight loss. Three good quality trials $[36,38]$ comparing combined nutrition and exercise interventions with standard post-partum care reported inconsistent findings; four of five fair-to-good quality RCTs reported small to modest weight loss $(0.6 \mathrm{~kg}$ to 
Table 2 Effect of postpartum nutrition and exercise interventions on maternal weight, adiposity

\begin{tabular}{|c|c|c|c|}
\hline Author, yr & Weight (kg) SD & BMI $\left(\mathrm{kg} / \mathrm{m}^{2}\right)$ & Adiposity \\
\hline \multicolumn{4}{|c|}{ Nutrition and Exercise interventions } \\
\hline \multirow[t]{10}{*}{ Bertz (2012) [30] } & Weight (12 weeks) & & Fat mass: \\
\hline & D: $-8.3 \pm 3.0$ & D: $-2.9 \pm 1.5$ & D: $-6.9 \pm 3.4$ \\
\hline & $E:-2.4 \pm 3.2$ & $\mathrm{E}:-0.8 \pm 1.0$ & $\mathrm{E}:-1.8 \pm 3.0$ \\
\hline & DE: $-6.9 \pm 3.0$ & DE: -2.51 .0 & DE: $-6.2 \pm 3.1$ \\
\hline & $C:-0.8 \pm 3.0 ; P<0.001$ & $C:-0.31 .1 ; P<0.001$ & $C:-0.7 \pm 3.1$ \\
\hline & Weight (1 year): & & 1 year: \\
\hline & D: $-10.2 \pm 5.7$ & D: $-3.6 \pm 2.0$ & D: $-9.2 \pm 5.6$ \\
\hline & $\mathrm{E}:-2.7 \pm 5.9$ & $\mathrm{E}:-0.9 \pm 2.0$ & $\mathrm{E}:-2.5 \pm 5.9$ \\
\hline & DE: $-7.3 \pm 6.3$ & DE: $-2.6 \pm 2.2$ & DE: $-6.0 \pm 7.0$ \\
\hline & $C:-0.9 \pm 6.6 ; P<0.001$ & $C:-0.3 \pm 2.4 ; P<0.001$ & $C:-1.8 \pm 6.2$ \\
\hline \multirow[t]{3}{*}{ Colleran (2012) [32] } & l: -5.8 (SE:3.5) & I: 0.9 (SE 1.5) & \\
\hline & C: -1.6 (SE:5.4); & C: 2.1 (SE1.4); & \\
\hline & $P<0.01$ & $P<0.01$ & \\
\hline \multirow[t]{3}{*}{ Leermakers (1998) [31] } & l: $-7.8 \pm 4.5$ & & \\
\hline & $C:-4.9 \pm 5.4$ & & \\
\hline & $\left(P^{a}=0.03\right)$ & & \\
\hline \multirow[t]{3}{*}{ Lovelady (2000) [37] } & I: $-4.8 \pm 1.7$ & I: $-1.8 \pm 0.6$ & Skinfold thickness; all 6 measures $(P<0.01)^{b}$ \\
\hline & $C:-0.8 \pm 2.3$ & $C:-0.3 \pm 0.9(P<0.01)$ & \\
\hline & $(P<0.001)$ & & \\
\hline \multirow[t]{4}{*}{ O'Toole (2003) [36] } & l: -7.3 & & \\
\hline & $C:-1.3$ & & \\
\hline & $(P<0.05)$ & & \\
\hline & SD-NR & & \\
\hline \multirow[t]{3}{*}{ Ostbye (2009) [29] } & l: $-0.9 \pm 5.1$ & & \\
\hline & $C:-0.36 \pm 4.9$ & & \\
\hline & $(P=0.25)$ & & \\
\hline \multirow[t]{8}{*}{ Craigie (2011) [28] } & l: $-1.6 \pm 2.0$ & & Waist circumference \\
\hline & $C: 0.2 \pm 2.2$ & & l: $-4.4 \pm 3.5$ \\
\hline & $(P=0.018)$ & & $C:-2.8 \pm 4.5$ \\
\hline & & & $(P=0.23)$ \\
\hline & & & $\%$ body fat \\
\hline & & & l: $-1.5 \pm 0.8$ \\
\hline & & & $C:-0.5 \pm 1.4$ \\
\hline & & & $(P=0.32)$ \\
\hline \multirow[t]{4}{*}{ Huang (2011) [25] } & I: -11.21 & & \\
\hline & $C:-11.04$ & & \\
\hline & (P-value NR) & & \\
\hline & SD-NR & & \\
\hline \multirow[t]{5}{*}{ Davenport (2011) [33] } & l: $-5.0 \pm 2.9$ & l: -1.8 & Waist-to-Hip Ratio \\
\hline & Diet + moderate intensity exercise; & moderate intensity & I: -0.05 \\
\hline & & l: -1.5 & l: -0.03 \\
\hline & I: $-4.2 \pm 4.0$ & low intensity & C: 0.0 \\
\hline & Diet + low intensity exercise; & $C: 0.0$ & SD:NR \\
\hline
\end{tabular}


Table 2 Effect of postpartum nutrition and exercise interventions on maternal weight, adiposity (Continued)

$\begin{array}{lll}\text { Historical controls: } & \text { SD-NR } & (P<0.05)^{\mathrm{a}} \\ -0.1 \pm 3.3 & (P<0.05)^{\mathrm{a}} & \\ (P<0.01)^{\mathrm{a}} & & \end{array}$

Exercise-only interventions

$\begin{array}{ll}\text { Dewey (1994) [34] } & \text { I: }-1.6 \\ & C:-1.6 \\ & (p>0.05) \\ & \text { SD-NR } \\ \text { Zourladani (2011) [27] } & \text { I: }-3.3 \\ & C:-1.3 \\ & (P=0.667) \\ & \text { SD-NR } \\ \text { Maturi (2011) [26] }-2.1 \\ & C: 0 \\ & (P<0.001) \\ & \text { SD-NR }\end{array}$

$\begin{array}{ll}\text { I: }-0.81 & \text { Waist-to-Hip Ratio } \\ \text { C: } 0.1 & \text { I: }-0.02 \\ (P<0.001) & C: 0.01 \\ \text { SD-NR } & (P=0.02) \\ & \text { SD-NR }\end{array}$

Diet-only interventions

$\begin{array}{lll}\text { Krummel (2010) [35] } & \text { I: }-2.9 \pm 11.8 & \text { I: }-0.54 \pm 1.9 \\ & \text { C: }-2.9 \pm 10.7 & C:-0.54 \pm 1.8(P>0.05) \\ & (P>0.05) & \end{array}$

$\mathrm{SD}=$ Standard Deviation; NR $=$ Not reported ${ }^{\text {a }}$-values reflect relationship between control and the medium and low intensity exercise groups. ${ }^{\mathrm{b}}$ Skinfold thickness measures include triceps, subscapular area, mid-axillary line, abdomen, thigh and suprailiac area.

$4 \mathrm{~kg}$ ) among post-partum women with the use of combined nutrition and exercise interventions. Evidence from three exercise-only RCTs is inconclusive. Evidence for exclusively dietary interventions on weight loss is insufficient. Additionally, evidence for the effect of post- partum lifestyle interventions on maternal adiposity, cardio-metabolic outcomes and biological markers is insufficient. Based on the number and quality of studies reviewed, we conclude that combined nutrition and exercise intervention can achieve post-partum weight loss,

Table 3 Strength of evidence for effect of postpartum lifestyle interventions on weight, adiposity, metabolic and biological markers

\begin{tabular}{|c|c|c|c|}
\hline Outcome & $\begin{array}{l}\text { Strength of } \\
\text { evidence }\end{array}$ & Intervention & Conclusions \\
\hline \multirow[t]{3}{*}{ Weight } & Low & Nutrition and Exercise & $\begin{array}{l}\text { Four good quality RCTs had inconsistent findings. Three of the four fair-to-good quality } \\
\text { studies reported greater weight loss in the intervention group compared to standard } \\
\text { postpartum care, but the RCTs were short ( } \leq 9 \text { months) in duration and had limited } \\
\text { generalizability to racial and ethnic minority groups. }\end{array}$ \\
\hline & Low & Exercise only & $\begin{array}{l}\text { Results were inconclusive for comparison of exercise-only interventions with standard } \\
\text { postpartum care, with low risk for bias, but moderate imprecision and inconsistency in } \\
\text { the dire study findings. }\end{array}$ \\
\hline & Insufficient & Nutrition only & $\begin{array}{l}\text { Only one RCT for comparison of diet-only with standard postpartum care, with high } \\
\text { attrition rates and differential loss to follow-up between treatment groups. }\end{array}$ \\
\hline Adiposity & Low & All interventions & $\begin{array}{l}\text { Few studies included adiposity and there is inconsistency in adiposity measures across } \\
\text { studies. One comparison showed a reduction in skinfold thickness; two comparisons } \\
\text { reported a statistically significant reduction in waist-to-hip ratios, but estimate of effect } \\
\text { were imprecise due to small sample sizes. }\end{array}$ \\
\hline Cardio-metabolic & Insufficient & All interventions & $\begin{array}{l}\text { One RCT compared lipid and glucose levels between women receiving a nutrition and } \\
\text { exercise intervention and historical controls }\end{array}$ \\
\hline Biological markers & Insufficient & All interventions & $\begin{array}{l}\text { Only one RCT was included for the outcome of adiponectin. Findings from the intervention } \\
\text { group were compared to non-randomized historical controls. }\end{array}$ \\
\hline
\end{tabular}


but we are unable to draw substantial conclusions of effectiveness due to the inconsistency in findings from the three higher quality RCTs $[20,36,38]$ and particularly the lack of differences in weight reported by the largest trial to date by Ostbye and colleagues [38].

This systematic review adds to the literature on postpartum weight management interventions in several relevant areas. First, we include a broader scope of studies that included four additional RCTs (two additional combined interventions, one exercise-only and one nutrition-only). Second, we expanded the clinical outcomes of the review to include important cardio-metabolic health outcomes and obesity-related biomarkers shown to be responsive to modifications in nutritional intake and exercise in earlier studies. Post-partum interventions that promote a favorable biomarker profile may reduce the risk of chronic obesity and the downstream consequences of cardiovascular disease, diabetes and obesity-related cancers (e.g. breast, endometrial, colon). Also we included a review of potential harms related to post-partum behavioral interventions. Some investigations have suggested that maternal weight loss can diminish the quality of lactation, but based on limited available data in the clinical trials reviewed [34], we found no evidence for an increase in adverse maternal events. We found no evidence for an increase in adverse maternal or infant outcomes.

There are few evidence-based guidelines for postpartum nutrition and exercise (Table 4). In 2009, the American Congress of Obstetricians and Gynecologists reaffirmed earlier recommendations for exercise in the post-partum period and advocated that pre-pregnancy exercise may be gradually resumed as soon as medically safe [39]. Guidelines from the Canadian Society for Exercise Physiologists [40] suggest that most women can return to their normal exercise programs after receiving medical clearance at their first postnatal check-up [30]. In the United Kingdom, guidelines governing dietary interventions and physical activity interventions for weight management after pregnancy were developed by the National Institute for Health and Clinical Excellence (NICE) [41]. These guidelines were developed based on a review of the efficacy cost-effectiveness of interventions,

Table 4 Comparison of National Institute of Clinical Excellence and American Congress of Obstetricians and Gynecologists Recommendations

\section{All Post-partum Women}

NICE 6-8-week postnatal check

In women who are overweight, obese or who have concerns about their weight, ask if they would like any further advice and support now - or later. If yes, make an appointment within the next 6 months for advice and support.

Provide current advice about how to lose weight after childbirth.

Ensure women have a realistic expectation of the time it will take to lose weight gained during pregnancy.

Discuss benefits of a healthy diet and regular physical activity,

Advice on healthy eating and physical activity should be tailored to individual patient circumstances.

Advise women to seek information from reliable sources

Provide details of appropriate community-based services

Encourage women to breastfeed.

Provide advice on recreational exercise from the Royal College of Obstetrics and Gynecology:

A mild exercise program of walking and stretching may begin immediately; after complicated deliveries, or lower segment caesareans, a medical provider should be consulted before resuming pre-pregnancy levels of physical activity.

Emphasize importance of physical activities that can be built into daily life.

ACOG Rapid return to pre-pregnancy activities is acceptable after an uncomplicated pregnancy and delivery

Moderate weight reduction after delivery does not interfere with lactation or neonatal weight

Postpartum exercise may help to reduce postpartum depression symptoms

Refer to weight specialist before the next pregnancy

Discuss healthy lifestyle behaviors at each visit.
Post-partum women with $\mathrm{BMI} \geq 30$

6-8 week postnatal check

Explain the increased risks that being obese poses to them and, if they become pregnant again, their unborn child.

Encourage them to lose weight.

Offer a structured weight-loss program or a referral to a dietitian or an appropriately trained health professional.

Provide Women who are not yet ready to lose weight with information about where they can get support when they are ready.

Use evidence-based behavior change techniques to motivate and support women to lose weight.

Encourage breastfeeding

No recommendations based on BMl 
fieldwork data and comments from stakeholders and experts and include targeted goals, such as a low-fat diet, regular physical activity, and identifying and addressing barriers to change.

There are limitations that deserve attention. Limitations to the current review include possible publication bias. Large-scale studies that showed no differences between groups can remain unpublished and affect the ability to draw meaningful conclusions. Also, we only included articles written in English which may have contributed to reporting bias. Additionally, few measures of maternal adiposity or cardio-metabolic outcomes were included in two or more studies. There were several fair quality trials with limited information on steps to ensure adherence to the intervention protocol. This limitation in intervention fidelity further reduces the overall strength of available evidence. There were little to no data reported on which components of the intervention were most often used by participants and which components were most associated with the desired outcome. Additionally, the extent or type of care provided to the comparator group varied between studies making it difficult to draw meaningful conclusions from some studies. Trials compared the intervention group to standard post-partum care, self-directed groups and control conditions in which no detailed descriptions were provided. Only three trials $[35,36,38]$ included an intervention that was longer than 6 months in duration, limiting the ability to assess the effects of interventions on long-term weight loss and other health outcomes. There was little data on potential harms of interventions, such as adverse clinical outcomes or participant time and effort.

\section{Conclusion}

This review has several relevant implications for clinical practice and future research. Although limited, obstetricians and primary care providers now have evidence to support referral to counseling and behavioral interventions for overweight and obese women after delivery. Providers will need to communicate these findings to patients, create partnerships with programs and facilities offering behavioral interventions, and facilitate timely referral of their patients to these programs. In this fashion, post-partum women can receive these interventions in collaborative, multidisciplinary settings. Future studies that include a larger sample size and are adequately powered to detect meaningful differences in weight between treatment and control groups, consistent outcome measures of maternal adiposity as well as levels of glucose and obesity-related biomarkers are needed to broaden our knowledge of effectiveness and potential harms of postpartum nutrition and exercise interventions. Studies with well-developed protocols to ensure intervention fidelity can provide important information on the effectiveness of individual intervention components. Future studies should include interventions lasting 9 months or more to provide data on longer term outcomes. Finally, studies should collect and report data on any adverse events of post-partum weight management. On-going RCTs registered with ClinicalTrials.gov [42] may provide additional insight on the effect of behavioral interventions on weight and relevant metabolic biomarkers.

\section{Additional files}

Additional file 1: Search strategy.

Additional file 2: PRISMA checklist.

\section{Abbreviations}

BMI: Body mass index; GRADE: The grading of recommendations assessment, development and evaluation; HbA1C: Hemoglobin A1C; HDL-C: High-density lipoprotein cholesterol; LDL-C: Low-density lipoprotein cholesterol; NICE: National institute for health and clinical excellence; RCT: Randomized controlled trials.

\section{Competing interests}

The authors declare that they have no competing of interests.

\section{Authors' contributions}

$A B$ was involved in the conceptual framework of the study. He was a primary reviewer of the studies included in the systematic review and was a primary writer. WN contributed to the conceptual framework and the protocol for the systematic review. RPU contributed to interpretation of study results and provided revisions to the text of the manuscript. All authors read and approved the final manuscript.

\section{Acknowledgements}

We would like to thank Dr. Russell Harris for comments on the protocol, interpretation of results and edits on the manuscript. Also, we are grateful to Dr. Aileen Jenna Beckam for her comments on the manuscript.

This paper reports on an independent study. Dr. Nicholson has funding by the National Institute for Diabetes and Digestive and Kidney Diseases (1R21DK095189-01A1). However, the agency had no role in the conduct of this study.

The paper was presented, in part, at the 30th Annual Meeting of The Obesity Society, San Antonio Texas, September 22, 2012 and the Annual Meeting of the Society for Pediatric and Perinatal Epidemiology Research, Minneapolis, Minnesota, June 23, 2012.

\section{Author details}

${ }^{1}$ Public Health Leadership Program, University of North Carolina Gillings Global School of Public Health, 135 Dauer Drive, Chapel Hill, NC, USA. ${ }^{2}$ Department of Obstetrics and Gynecology, University of North Carolina School of Medicine, 321 South Columbia Street, Chapel Hill, NC, USA. 3Department of Obstetrics \& Gynecology, Division of Women's Primary Health, 3027 Old Clinic Building, CB \# 7570, Chapel Hill, NC, USA. ${ }^{4}$ Diabetes and Obesity Core, Center for Women's Health Research, University of North Carolina School of Medicine, 3027 Old Clinic Building, CB \# 7570, Chapel Hill, NC, USA.

Received: 10 December 2013 Accepted: 17 August 2014

Published: 10 September 2014

\section{References}

1. Flegal KM, Carroll MD, Kit BK, Ogden CL: Prevalence of obesity and trends in the distribution of body mass index among US adults, 1999-2010. JAMA 2012, 307(5):491-497.

2. Gunderson EP: Gestational diabetes and nutritional recommendations. Curr Diab Rep 2004, 4(5):377-386.

3. Gore SA, Brown DM, West DS: The role of postpartum weight retention in obesity among women: a review of the evidence. Ann Behav Med 2003, 26(2):149-159. 
4. Rooney BL, Schauberger CW, Mathiason MA: Impact of perinatal weight change on long-term obesity and obesity-related illnesses. Obstet Gynecol 2005, 106(6):1349-1356.

5. Kral JG: Preventing and treating obesity in girls and young women to curb the epidemic. Obes Res 2004, 12(10):1539-1546.

6. Smith DE, Lewis CE, Caveny JL, Perkins LL, Burke GL, Bild DE: Longitudinal changes in adiposity associated with pregnancy. The CARDIA study. coronary artery risk development in young adults study. JAMA 1994, 271(22):1747-1751.

7. Appel LJ, Champagne CM, Harsha DW, Cooper LS, Obarzanek E, Elmer PJ, Stevens VJ, Vollmer WM, Lin PH, Svetkey LP, Stedman SW, Young DR: Effects of comprehensive lifestyle modification on blood pressure control: main results of the PREMIER clinical trial. JAMA 2003, 289(16):2083-2093.

8. Knowler WC, Barrett-Connor E, Fowler SE, Hamman RF, Lachin JM, Walker EA, Nathan DM: Diabetes Prevention Program Research G. Reduction in the incidence of type 2 diabetes with lifestyle intervention or metformin. N Engl J Med 2002, 346(6):393-403.

9. Steinberg DM, Tate DF, Bennett GG, Ennett S, Samuel-Hodge C, Ward DS: Daily self-weighing and adverse psychological outcomes: a randomized controlled trial. Am J Prev Med 2014, 46(1):24-29.

10. Mari G, Deprest J, Schenone M, Jackson S, Samson J, Brocato B, Tate D, Sullivan R, White G, Dhanireddy R, Mandrell T, Gupta S, Skobowjat C, Slominski A, Cohen HL, Schlabritz-Loutsevitch N: A novel translational model of percutaneous fetoscopic endoluminal tracheal occlusion baboons (Papio spp.). Fetal Diagn Ther 2014, 35(2):92-100.

11. Bombardier CH, Fann JR, Wilson CS, Heinemann AW, Richards JS, Warren AM, Brooks L, Warms CA, Temkin NR, Tate DG: A randomized controlled trial of venlafaxine XR for major depressive disorder after spinal cord injury: methods and lessons learned. J Spinal Cord Med 2014, 37(3):247-263.

12. Artal R, OToole M: Guidelines of the American College of Obstetricians and Gynecologists for exercise during pregnancy and the postpartum period. Br J Sports Med 2003, 37(1):6-12. discussion 12.

13. van der Pligt $P$, Willcox J, Hesketh KD, Ball K, Wilkinson S, Crawford D, Campbell K: Systematic review of lifestyle interventions to limit postpartum weight retention: implications for future opportunities to prevent maternal overweight and obesity following childbirth. Obes Rev 2013, 14(10):792-805.

14. Sesso HD, Wang L, Buring JE, Ridker PM, Gaziano JM: Comparison of interleukin- 6 and C-reactive protein for the risk of developing hypertension in women. Hypertension 2007, 49(2):304-310.

15. Schmidt MI, Duncan BB, Sharrett AR, Lindberg G, Savage PJ, Offenbacher S Azambuja MI, Tracy RP, Heiss G: Markers of inflammation and prediction of diabetes mellitus in adults (Atherosclerosis Risk in Communities study): a cohort study. Lancet 1999, 353(9165):1649-1652.

16. Pradhan AD, Cook NR, Buring JE, Manson JE, Ridker PM: C-reactive protein is independently associated with fasting insulin in nondiabetic women. Arterioscler Thromb Vasc Biol 2003, 23(4):650-655.

17. Liu S, Manson JE, Buring JE, Stampfer MJ, Willett WC, Ridker PM: Relation between a diet with a high glycemic load and plasma concentrations of high-sensitivity C-reactive protein in middle-aged women. Am J Clin Nutr 2002, 75(3):492-498.

18. Cseh K, Baranyi E, Melczer Z, Csakany GM, Speer G, Kovacs M, Gero G, Karadi I, Winkler G: The pathophysiological influence of leptin and the tumor necrosis factor system on maternal insulin resistance: negative correlation with anthropometric parameters of neonates in gestational diabetes. Gynecol Endocrinol 2002, 16(6):453-460.

19. Kirwan JP, Hauguel-De Mouzon S, Lepercq J, Challier JC, Huston-Presley L, Friedman JE, Kalhan SC, Catalano PM: TNF-alpha is a predictor of insulin resistance in human pregnancy. Diabetes 2002, 51(7):2207-2213.

20. IImonen J, Isolauri E, Poussa T, Laitinen K: Impact of dietary counselling and probiotic intervention on maternal anthropometric measurements during and after pregnancy: a randomized placebo-controlled trial. Clin Nutr 2011, 30(2):156-164.

21. Harris RPHM, Woolf SH, Lohr K, Mulrow CD, Teutsch SM, Atkins D: Current methods of the US preventive services task force: a review of the process. Am J Prev Med 2001, 20(3 Suppl):21-35.

22. Atkins DBD, Briss PA, Eccles M, Falck-Ytter Y, Flottorp S, Guyatt GH, Harbour RT, Haugh MC, Henry D, Hill S, Jaeschke R, Leng G, Liberati A, Magrini N, Mason J, Middleton P, Mrukowicz J, O'Connell D, Oxman AD, Phillips B, Schünemann HJ, Edejer T, Varonen H, Vist GE, Williams JW Jr, Zaza S:
Grading quality of evidence and strength of recommendations. BMJ 2004, 328(7454):1490.

23. Centre for Reviews and Dissemination: Systematic Reviews in Centre for Reviews and Dissemination's guidance for undertaking reviews in healthcare. York, England: 2009.

24. Guide for Effectiveness and Comparative Effectiveness Reviews. AHRQ Publication No. 10(11)-EHC063-EF. Rockville, MD: Agency for Healthcare Research and Quality; 2011. Chapters available at: www.effectivehealthcare.ahrq.gov.

25. Huang TTYC, Tsai YC: A diet and physical activity intervention for preventing weight retention among Taiwanese childbearing women: a randomized controlled trial. Midwifery 2011, 27(2):257-264.

26. Maturi SMAP, Abedi P: Effect of physical activity intervention based on a pedometer on physical activity level and anthropometric measures after childbirth: a randomized controlled trial. BMC Pregnancy Childbirth 2011, 11(10):103.

27. Zourladani A, Tsaloglidou A, Tzetzis G, Tsorbatzoudis C, Matziari C: The effect of a low impact exercise training programme on the well-being of Greek postpartum women: A randomised controlled trial. Int SportMed J 2001, 12(1):30-38.

28. Craigie AMMM, Barton KL, Treweek S, Anderson AS: Supporting postpartum weight loss in women living in deprived communities: design implications for a randomized control trial. Eur J Clin Nutr 2011, 65:952-958.

29. Ostbye T, Krause KM, Lovelady CA, Morey MC, Bastian LA, Peterson BL, Swamy GK, Brouwer RJ, McBride CM: Active mothers postpartum: a randomized controlled weight-loss intervention trial. Am J Prev Med 2009, 37(3):173-180

30. Bertz F, Brekke HK, Ellegard L, Rasmussen KM, Wennergren M, Winkvist A: Diet and exercise weight-loss trial in lactating overweight and obese women. Am J Clin Nutr 2012, 96(4):698-705.

31. Leermakers EAAK, Wing RR: Reducing postpartum weight retention through a correspondence intervention. Int J Obes Relat Metab Disord 1998, 22:1103-1109.

32. Colleran HL, Lovelady CA: Use of mypyramid menu planner for moms in a weight-loss intervention during lactation. J Acad Nutr Diet 2012, 112(4):553-558

33. Davenport MHGl, Sopper MM, Mottola MF: Postpartum exercise regardless of intensity improves chronic disease risk factors. Med Sci Sports Exerc 2011, 43(6):951-958.

34. Dewey KGLC, Nommsen-Rivers LA, McCory MA, Lonnerdal B: A randomized study of the effects of aerobic exercise by lactating women on breastmilk volume and composition. N Engl J Med 1994, 330:449-453.

35. Krummel D, Semmens E, MacBride AM, Fisher B: Lessons learned from the mothers' overweight management study in 4 West Virginia WIC offices... women, infants, and children. J Nutr Educ Behav 2010, 42(3S):S52-S58.

36. OToole ML, Sawicki MA, Artal R: Structured diet and physical activity prevent postpartum weight retention. J Women's Health (15409996) 2003, 12(10):991-998.

37. Lovelady CAGK, Moreno KL, Williams JP: The effect of weight loss in overweight, lactating women on the growth of their infants. N Engl J Med 2000, 342:449-453.

38. Ostbye TKK, Lovelady CA, Morey MC, Bastian LA, Peterson BL, Swamy GK, Brower RJ, McBride CM: Active mothers postpartum. A randomized controlled weight-loss intervention trial. Am J Prev Med 2009, 7(30):173.

39. American Congress of Obstetricians and Gynecologists Practice Bulletin: Exercise during pregnancy and the postpartum period. ACOG Committee opinion. Number 267. Obstet Gynecol 2002, 99(6):975-991.

40. Davies GAWL, Mottola MF, MacKinnon C: Joint SOGC/CSEP clinical practice guideline: exercise in pregnancy and the postpartum period. Can J Appl Physiol 2003, 28:330-341.

41. National Institute for Health and Clinical Excellence (NICE): Weight Management Before, During and After Pregnancy. In NICE Public Health Guidance 27. London, UK: 2010.

42. Clinical Trials Registry. http://www.clinicaltrials.gov.

\section{doi:10.1186/1471-2393-14-319}

Cite this article as: Berger et al:: Systematic review of the effect of individual and combined nutrition and exercise interventions on weight, adiposity and metabolic outcomes after delivery: evidence for developing behavioral guidelines for post-partum weight control. BMC Pregnancy and Childbirth 2014 14:319. 